\title{
SOME REGULARITY RESULTS FOR THE PSEUDOSPECTRAL ABSCISSA AND PSEUDOSPECTRAL RADIUS OF A MATRIX*
}

\author{
MERT GÜRBÜZBALABAN ${ }^{\dagger}$ AND MICHAEL L. OVERTON ${ }^{\dagger}$
}

\begin{abstract}
The $\varepsilon$-pseudospectral abscissa $\alpha_{\varepsilon}$ and radius $\rho_{\varepsilon}$ of an $n \times n$ matrix are, respectively, the maximal real part and the maximal modulus of points in its $\varepsilon$-pseudospectrum, defined using the spectral norm. It was proved in [A.S. Lewis and C.H.J. Pang, SIAM J. Optim., 19 (2008), pp. 1048-1072] that for fixed $\varepsilon>0, \alpha_{\varepsilon}$ and $\rho_{\varepsilon}$ are Lipschitz continuous at a matrix $A$ except when $\alpha_{\varepsilon}$ and $\rho_{\varepsilon}$ are attained at a critical point of the norm of the resolvent (in the nonsmooth sense), and it was conjectured that the points where $\alpha_{\varepsilon}$ and $\rho_{\varepsilon}$ are attained are not resolvent-critical. We prove this conjecture, which leads to the new result that $\alpha_{\varepsilon}$ and $\rho_{\varepsilon}$ are Lipschitz continuous, and also establishes the Aubin property with respect to both $\varepsilon$ and $A$ of the $\varepsilon$-pseudospectrum for the points $z \in \mathbb{C}$ where $\alpha_{\varepsilon}$ and $\rho_{\varepsilon}$ are attained. Finally, we give a proof showing that the pseudospectrum can never be "pointed outwards."
\end{abstract}

Key words. pseudospectrum, pseudospectral abscissa, pseudospectral radius, eigenvalue perturbation, Lipschitz multifunction, Aubin property

AMS subject classifications. Primary, 15A18, 93D09, 49J52; Secondary, 65K10, 90C26

DOI. $10.1137 / 110822840$

1. Introduction. Let $\|\cdot\|$ denote the vector or matrix 2-norm (spectral norm). For real $\varepsilon \geq 0$, the $\varepsilon$-pseudospectrum of a matrix $A \in \mathbb{C}^{n \times n}$ [TE05] is the union of the spectra of nearby matrices,

$$
\Lambda_{\varepsilon}(A)=\left\{z \in \mathbb{C}: z \in \Lambda(A+E) \text { for some } E \in \mathbb{C}^{n \times n} \text { with }\|E\| \leq \varepsilon\right\},
$$

where $\Lambda(A)$ denotes the spectrum (set of eigenvalues) of $A$. Equivalently, $\Lambda_{\varepsilon}$ is the upper level set of the norm of the resolvent of $A-z I$,

$$
\Lambda_{\varepsilon}(A)=\left\{z:\left\|(A-z I)^{-1}\right\| \geq \frac{1}{\varepsilon}\right\}
$$

and the lower level set of the smallest singular value of $A-z I$,

$$
\Lambda_{\varepsilon}(A)=\left\{z \in \mathbb{C}: \sigma_{n}(A-z I) \leq \varepsilon\right\} .
$$

The $\varepsilon$-pseudospectral abscissa of $A$ is the largest of the real parts of the elements of the pseudospectrum, i.e.,

$$
\alpha_{\varepsilon}(A)=\max \left\{\operatorname{Re} z: z \in \Lambda_{\varepsilon}(A)\right\} .
$$

The case $\varepsilon=0$ reduces to the spectral abscissa $\alpha(A)$, which measures the growth or decay of solutions to the continuous-time dynamical system $\dot{x}=A x$; in particular, $\alpha(A)$ is negative if and only if the solution decays to zero for all initial states. For $\varepsilon>0$, the pseudospectral abscissa of $A$ characterizes asymptotic behavior when $A$

\footnotetext{
* Received by the editors January 31, 2011; accepted for publication (in revised form) January 18, 2012; published electronically April 4, 2012.

http://www.siam.org/journals/siopt/22-2/82284.html

$\dagger$ Courant Institute of Mathematical Sciences, New York University, 251 Mercer Street, New York, NY 10012 (mert@cims.nyu.edu, overton@cs.nyu.edu). This work was supported in part by the National Science Foundation under grant DMS-1016325.
}

281 
is subject to perturbations bounded in norm by $\varepsilon$. Furthermore, as $\varepsilon$ varies from 0 to $\infty$, the map $\alpha_{\varepsilon}$ ranges from measuring asymptotic behavior to measuring initial behavior of the solutions to $\dot{x}=A x$ [BLO03, p. 86].

The analogous measure for discrete-time systems $x_{k+1}=A x_{k}$ is the $\varepsilon$-pseudospectral radius

$$
\rho_{\varepsilon}(A)=\max \left\{|z|: z \in \Lambda_{\varepsilon}(A)\right\} .
$$

The case $\varepsilon=0$ reduces to $\rho(A)$, the spectral radius of $A$, which is less than one if and only if solutions decay to zero for all initial states.

Below, we will refer to points where $\alpha_{\varepsilon}$ or $\rho_{\varepsilon}$ is attained. By this we mean the points $z \in \Lambda_{\varepsilon}$ where the real part or the modulus, respectively, is maximized.

For fixed $\varepsilon, \Lambda_{\varepsilon}: A \rightarrow \Lambda_{\varepsilon}(A)$ is continuous [Kar03, Theorem 2.3.7]. Since $\Lambda_{\varepsilon}$ is set-valued, continuity is to be understood in the Hausdorff metric. Recently, Lewis and Pang [LP08] proved that $\Lambda_{\varepsilon}$ has further regularity properties. Specifically, they showed that $\Lambda_{\varepsilon}$ has a local Lipschitz property known as the Aubin property everywhere except at resolvent-critical points (to be defined in the next section). It was also proved that for fixed $\varepsilon>0, \alpha_{\varepsilon}$ (respectively, $\rho_{\varepsilon}$ ) is Lipschitz continuous at a matrix $A$ if the points where $\alpha_{\varepsilon}$ (respectively, $\rho_{\varepsilon}$ ) is attained are not resolvent-critical (a consequence of [LP08, Corollary 7.2 and Theorem 5.2]). The fact that for a fixed matrix $A$ the number of resolvent-critical points is finite leads to the property that $\Lambda_{\varepsilon}, \alpha_{\varepsilon}$, and $\rho_{\varepsilon}$ are Lipschitz around a given matrix $A$ for all but finitely many $\varepsilon>0$ [LP08, Corollary 8.5]. It was conjectured that the points where $\alpha_{\varepsilon}$ is attained are not resolvent-critical [LP08, Conjecture 8.9]. We prove this conjecture, which implies that for fixed $\varepsilon>0, \alpha_{\varepsilon}$ is locally Lipschitz continuous on $\mathbb{C}^{n \times n}$. Our proof also applies to $\rho_{\varepsilon}$, proving that it is also locally Lipschitz. We also prove the Aubin property of the $\varepsilon$-pseudospectrum with respect to both $\varepsilon$ and $A$ for the points $z \in \mathbb{C}$ where $\alpha_{\varepsilon}$ and $\rho_{\varepsilon}$ are attained. Finally, we give a proof showing that $\Lambda_{\varepsilon}$ can never be "pointed outwards."

2. Previous results and notation. Before stating the conjecture, we need the following known results and definitions from [LP08]. We write MSV : $M^{n} \rightrightarrows \mathbb{C}^{n} \times \mathbb{C}^{n}$, with

$$
\operatorname{MSV}(A):=\{(u, v) \mid u, v \text { minimal left and right singular vectors of } A\} .
$$

In this definition, $u, v$ are minimal left and right singular vectors of $A$ if they are unit vectors satisfying

$$
\sigma_{n}(A) u=A v \quad \text { and } \quad \sigma_{n}(A) v=A^{*} u,
$$

where $A^{*}$ is the Hermitian transpose of $A$. The set

$$
Y(A):=\left\{v^{*} u \mid(u, v) \in \operatorname{MSV}(A)\right\}
$$

will be a key tool in our analysis since, for a fixed $A$ and for $z \notin \Lambda(A)$, we have [LP08, Proposition 4.5]

$$
Y(A-z I)=\partial\left(-\sigma_{n}(A-z I)\right)
$$

where $\partial$ is the subdifferential in the sense of [RW98, Definition 8.3]. This leads to the following. 
Definition 2.1. A point $z \in \mathbb{C}$ is resolvent-critical for $A \in \mathbb{C}^{n \times n}$ if either $z \in \Lambda(A)$ or $0 \in Y(A-z I)$.

Thus, a resolvent-critical point is either an eigenvalue of $A$ or a critical point of the norm of the resolvent in the nonsmooth sense (see [LP08, Proposition 4.7 and Definition 4.8]).

Now we are ready to state Lewis and Pang's conjecture.

Conjecture 2.1 (see [LP08, Conjecture 8.9]). The points $z \in \Lambda_{\varepsilon}(A)$ where the pseudospectral abscissa $\alpha_{\varepsilon}(A)$ is attained are not resolvent-critical.

In the following, we will also need the Aubin property, a local Lipschitz property for set-valued mappings.

Definition 2.2 (see [RW98, Definition 9.36]). A mapping $S: \mathbb{R}^{n} \rightrightarrows \mathbb{R}^{m}$ has the Aubin property at $\bar{x}$ for $\bar{u}$, where $\bar{x} \in \mathbb{R}^{n}$ and $\bar{u} \in S(\bar{x})$, if gph $S$ is locally closed at $(\bar{x}, \bar{u})$ and there are neighborhoods $V$ of $\bar{x}$ and $W$ of $\bar{u}$, and a constant $\kappa \in \mathbb{R}_{+}$, such that

$$
S\left(x^{\prime}\right) \cap W \subset S(x)+\kappa\left|x^{\prime}-x\right| \mathbb{B} \quad \text { for all } x, x^{\prime} \in V,
$$

where $\mathbb{B}$ is the unit ball in $\mathbb{R}^{m}$.

3. New results. Let bd $\Lambda_{\varepsilon}(A)$ denote the boundary of the pseudospectrum of $A$. We now state our main result on the resolvent-critical points of bd $\Lambda_{\varepsilon}(A)$, which is based on a result in [ABBO10].

THEOREM 3.1. If $z \in \operatorname{bd} \Lambda_{\varepsilon}(A)$ is resolvent-critical for some $\varepsilon>0$ and a matrix $A$, then there exists an integer $m \geq 2, \tilde{\theta}$ real, and $\tilde{\rho}$ positive real such that for all $\omega<\pi / m, \Lambda_{\varepsilon}$ contains $m$ equally spaced circular sectors of angle at least $\omega$ centered at $z$, that is,

$$
\Lambda_{\varepsilon}(A) \supset\left\{z+\rho e^{i \theta} \mid \theta \in[\tilde{\theta}+2 \pi k / m-\omega / 2, \tilde{\theta}+2 \pi k / m+\omega / 2], \rho \leq \tilde{\rho}\right\}
$$

for all $k=0,1,2, \ldots, m-1$.

Proof. Assume that $z \in$ bd $\Lambda_{\varepsilon}(A)$ is resolvent-critical. Since $\varepsilon>0, z \notin \Lambda(A)$, so there exists a pair of singular vectors $(\tilde{u}, \tilde{v}) \in \operatorname{MSV}(A-z I)$ such that $\tilde{v}^{*} \tilde{u}=0$. If the smallest singular value of $A-z I$ is simple, then it follows from [ABBO10, Theorem 9 and subsequent discussion] that there exists an integer $m \geq 2$ such that for all $\omega<\pi / m, \Lambda_{\varepsilon}$ contains $m$ circular sectors of angle at least $\omega$ centered at $z$ as stated, and so the result is proved. If the smallest singular value of $A-z I$ is not simple, consider a perturbed matrix $A_{\delta}=A-\delta \tilde{u} \tilde{v}^{*}$ for $\delta \in(0, \varepsilon)$. Then, the smallest singular value of $A_{\delta}-z I$ is simple with value $\varepsilon-\delta$ and corresponding singular vectors $\tilde{u}, \tilde{v}$ with $\tilde{u}^{*} \tilde{v}=0$. Thus, we can apply [ABBO10, Theorem 9] to $A_{\delta}$, finding that for all $\omega<\pi / m, \Lambda_{\varepsilon-\delta}\left(A_{\delta}\right)$ contains $m \geq 2$ circular sectors of angle at least $\omega$ centered at $z$. But immediately from the definition, using the triangle inequality for the norm, we have (see Figure 3.1)

$$
\Lambda_{\varepsilon}(A) \supset \Lambda_{\varepsilon-\delta}\left(A_{\delta}\right)
$$

proving the result.

We conjecture that the only possible value for $m$ in Theorem 3.1 is 2 . See [ABBO10, Figure 3].

Clearly, at a point where the pseudospectral abscissa or pseudospectral radius is attained, the pseudospectrum cannot contain $m \geq 2$ circular sectors as defined above. As a consequence, we have the following. 

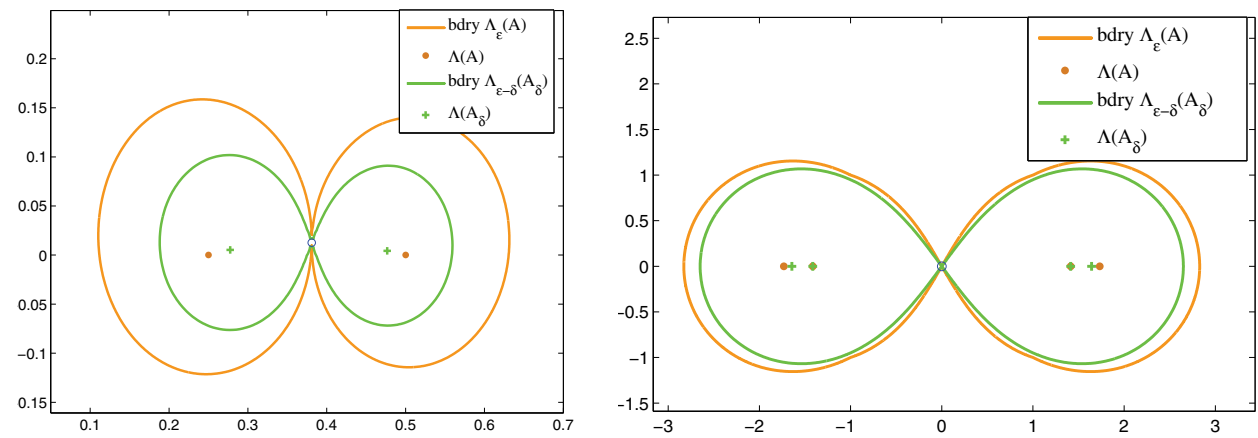

Fig. 3.1. Figure illustrates the inclusion $\Lambda_{\varepsilon-\delta}\left(A_{\delta}\right) \subset \Lambda_{\varepsilon}(A)$. On the left, $A$ is the $4 \times 4$ matrix with tangential coalescence given in [ABBO10, right panel of Figure 1] with $\varepsilon=0.0136$ and $\delta=0.005$. On the right, $A$ is the reverse diagonal matrix with entries 1, 1, 3, and 2 (Gracia's example), $\varepsilon=1$ and $\delta=0.1$. The smallest singular value of $A-z I$ has multiplicity 2 in both cases, and $m=2$ in both cases. The plots are obtained with the software package EigTool [Wri02].

Corollary 3.2. For any $\varepsilon>0$, the points where the pseudospectral abscissa $\alpha_{\varepsilon}$ or pseudospectral radius $\rho_{\varepsilon}$ is attained are not resolvent-critical.

Thus, Conjecture 2.1 is proved. Furthermore, Theorem 3.1 implies the following regularity results about $\alpha_{\varepsilon}, \rho_{\varepsilon}$, and $\Lambda_{\varepsilon}$.

Corollary 3.3. Let $\varepsilon>0$ be given, and let $z_{*} \in$ bd $\Lambda_{\varepsilon}\left(A_{*}\right)$ be a point where the pseudospectral abscissa $\alpha_{\varepsilon}\left(A_{*}\right)$ or pseudospectral radius $\rho_{\varepsilon}\left(A_{*}\right)$ is attained for some matrix $A_{*}$. Then, the map $A \rightarrow \Lambda_{\varepsilon}(A)$ has the Aubin property at $A_{*}$ for $z_{*}$.

Proof. By Corollary 3.2, $z_{*}$ is not resolvent-critical. The result follows from [LP08, Theorem 5.2].

COROLlary 3.4. For any fixed $\varepsilon>0, \alpha_{\varepsilon}$ and $\rho_{\varepsilon}$ are Lipschitz continuous at any matrix A.

Proof. Let $A \in \mathbb{C}^{n \times n}$ be given. By Corollary $3.3, \Lambda_{\varepsilon}$ has the Aubin property at $A$ at all the points where the pseudospectral abscissa or pseudospectral radius is attained. An application of [LP08, Corollary 7.2(a)] with $F=\Lambda_{\varepsilon}, g(x)=\operatorname{Re}(-x)$ proves the Lipschitz continuity of $\alpha_{\varepsilon}$ while using $F=\Lambda_{\varepsilon}, g(x)=-|x|$ proves the Lipschitz continuity of $\rho_{\varepsilon}$.

CoROLlaRY 3.5. Let $z_{*} \in \mathbb{C}$ be a point where the pseudospectral abscissa $\alpha_{\varepsilon_{*}}(A)$ or pseudospectral radius $\rho_{\varepsilon_{*}}(A)$ is attained for some $\varepsilon_{*}>0$ and $A \in \mathbb{C}^{n \times n}$. Then the map $\varepsilon \rightarrow \Lambda_{\varepsilon}(A)$ has the Aubin property at $\varepsilon_{*}$ for $z_{*}$.

Proof. From (2.1) and Corollary 3.2, we have $0 \notin Y\left(A-z_{*} I\right)=\partial\left(-\sigma_{n}\left(A-z_{*} I\right)\right)$. The result then follows from [LP08, Proposition 5.3], using, as is done there, the inclusion $-\partial\left(\sigma_{n}(A-z I)\right) \subset \partial\left(-\sigma_{n}(A-z I)\right)$.

In the terminology of [BLO03, Definition 4.5 and its corrigendum], a point $z$ is called nondegenerate with respect to $\Lambda_{\varepsilon}(A)$ if $Y(A-z I) \neq\{0\}$. Thus, Corollary 3.2 implies that a point where the pseudospectral abscissa or pseudospectral radius is attained is nondegenerate. This leads to the following generalization of [BLO03, Proposition 4.8].

Proposition 3.6. Let $z_{*}$ be a point where $\alpha_{\varepsilon}(A)$ or $\rho_{\varepsilon}(A)$ is attained for some $\varepsilon>0$ and a matrix $A$. Then the boundary of $\Lambda_{\varepsilon}(A)$ is differentiable at $z_{*} ;$ i.e., the boundary of $\Lambda_{\varepsilon}(A)$ around $z_{*}$ is a curve that is differentiable at $z_{*}$.

Proof. This follows from [BLO03, Proposition 4.8] and the fact that $z_{*}$ is nondegenerate. 
It was proved in [BLO03, Proposition 4.8] that the pseudospectrum cannot be "pointed outwards" at nondegenerate points. By this, one means that around a nondegenerate point $z_{*}$, the pseudospectrum can never be contained in a circular sector of angle strictly less than $\pi$ centered at $z_{*}$. It was further stated that a more detailed analysis due to Trefethen shows that the pseudospectrum is never pointed outwards. Since the latter result, based on eigenvalue perturbation theory, was never published, we give a new proof here.

Proposition 3.7. Let $z_{*}$ be on the boundary of the pseudospectrum, i.e., $z_{*} \in$ $\operatorname{bd} \Lambda_{\varepsilon}(A)$ for some $\varepsilon>0$ and a matrix $A$. The pseudospectrum near $z_{*}$ cannot be contained in a circular sector of angle $<\pi$ centered at $z_{*}$; that is, for all $\omega \in[0, \pi)$, $\tilde{\theta} \in[0,2 \pi)$ and $\tilde{\rho}$ positive real, there exists a point $y \in \Lambda_{\epsilon}(A)$ such that we have $\left|y-z_{*}\right| \leq \tilde{\rho}$ but $y$ is not contained in the circular sector

$$
z_{*}+\left\{\rho e^{i \theta} \mid[\tilde{\theta}-\omega / 2, \tilde{\theta}+\omega / 2], \rho \leq \tilde{\rho}\right\} .
$$

Proof. If $z_{*}$ is nondegenerate, then the result follows from [BLO03, Proposition 4.7]. Otherwise, $z_{*}$ is resolvent-critical and the result follows from Theorem 3.1 .

\section{REFERENCES}

[ABBO10] R. Alam, S. Bora, R. Byers, And M. L. Overton, Characterization and construction of the nearest defective matrix via coalescence of pseudospectral components, Linear Algebra Appl., 435 (2011), pp. 494-513.

[BlO03] J. V. Burke, A. S. Lewis, And M. L. Overton, Optimization and pseudospectra, with applications to robust stability, SIAM J. Matrix Anal. Appl., 25 (2003), pp. 80-104; corrigendum available online at www.cs.nyu.edu/overton/ papers/pseudo_corrigendum.html.

[Kar03] M. Karow, Geometry of Spectral Value Sets, Ph.D. thesis, Universität Bremen, Bremen, Germany, 2003.

[LP08] A. S. LeWIS AND C. H. J. PANG, Variational analysis of pseudospectra, SIAM J. Optim., 19 (2008), pp. 1048-1072.

[RW98] R. T. Rockafellar and R. J. B. Wets, Variational Analysis, Springer, New York, 1998.

[Te05] L. N. Trefethen and M. Embree, Spectra and Pseudospectra: The Behavior of Nonnormal Matrices and Operators, Princeton University Press, Princeton, NJ, 2005.

[Wri02] T. G. Wright, EigTool: A Graphical Tool for Nonsymmetric Eigenproblems, http:// web.comlab.ox.ac.uk/pseudospectra/eigtool/ (2002).

Copyright $@$ by SIAM. Unauthorized reproduction of this article is prohibited. 